\title{
Evolution of bistable dynamics in spiking neural controllers for agents performing olfactory attraction and aversion
}

\author{
Nicolas Oros ${ }^{*}$, Volker Steuber, Neil Davey, Lola Cañamero, Rod Adams \\ From Nineteenth Annual Computational Neuroscience Meeting: CNS*2010 \\ San Antonio, TX, USA. 24-30 July 2010
}

Research on the olfactory system of Drosophila has shown that individual glomeruli mediate innate olfactory attraction and aversion [1]. The authors showed that increasing concentrations of vinegar result in excitation of an additional glomerulus, which can initiate a behavioral switch from attraction to aversion. This implies that high concentrations of vinegar activate a specific set of sensory neurons that is not activated by low concentrations.

In this research, we used a genetic algorithm to evolve spiking neural controllers for agents that were attracted by low concentrations of a simulated chemical but were repelled by high concentrations of the same chemical. We investigated how the evolved neural controllers could encode information about the chemical concentration to perform this task. We also wanted to see if the neural controllers needed to be equipped with different types of olfactory sensory neurons or if only one type was sufficient. We first performed experiments where the agent could use only one kind of sensors that responded to the whole range of concentrations. In a second set of experiments, the agents could use an additional pair of sensors only activated by high concentrations.

In our model, simulated agents could move in a $2 \mathrm{D}$ environment and the neural controller was placed on a 2D substrate. We used leaky integrate-and-fire neurons with temporal synaptic integration and transmission delays. The neural network was generated using a developmental model with an indirect encoding strategy that mapped an artificial genome onto the network connectivity.

Our model was able to evolve neural controllers with complex dynamics that used both neuronal firing rates and temporal coincidence to encode information about the olfactory environment. Surprisingly, the fittest evolved agent did not require an additional pair of sensors that responded only to high concentrations, different from what has been observed in Drosophila. A central feature that implemented the attraction and aversion behavior of the agent was the combination of bilateral inhibition and excitatory self-connections. This resulted in a cascade of two bi-stable systems, one of which was responsible for basic motor control and for attraction at low concentrations. The second bi-stable system controlled the switching between the states of the first bi-stable system when the concentration was too high.

These results show that an artificial life based approach to the study of natural processes like olfaction is feasible, and that it can suggest neural coding strategies that may be used by natural systems.

Published: 20 July 2010

\section{Reference}

1. Semmelhack JL, Wang JW: Select drosophila glomeruli mediate innate olfactory attraction and aversion. Nature 2009, 459:218-223.

doi:10.1186/1471-2202-11-S1-P92

Cite this article as: Oros et al:: Evolution of bistable dynamics in spiking neural controllers for agents performing olfactory attraction and aversion. BMC Neuroscience 2010 11(Suppl 1):P92.

\footnotetext{
* Correspondence: n.oros@herts.ac.uk

Science and Technology Research Institute, University of Hertfordshire, Hatfield, Hertfordshire, AL10 9AB, UK
} 